\title{
INDUCED STARBURST AND NUCLEAR ACTIVITY: FAITH, FACTS AND THEORY
}

\author{
Isaac Shlosman \\ Theoretical Astrophysics 130-33 \\ California Institute of Technology \\ Pasadena, CA 91125
}

\begin{abstract}
The problem of the origin of starburst and nuclear (nonstellar) activity in galaxies is reviewed. A physical understanding of mechanism(s) that induce both types of activity requires one to address the following issues: 1) what is the source of fuel that powers starbursts and active galactic nuclei? and 2) how it is channeled towards the central regions of host galaxies? As a possible clue, we examine the role of non-axisymmetric perturbations of galactic disks and analyze their potential triggers. Global gravitational instabilities in the gas on scales $\sim 100 \mathrm{pc}$ appear to be crucial for fueling the active galactic nuclei.
\end{abstract}

\section{Introduction}

The nuclear and circumnuclear regions of some galaxies exhibit profound activity. First, a high rate of massive star formation (hereafter SFR) has been detected in the inner kpc of "starburst" galaxies (SBGs), which appear to be disk galaxies, at least at low redshifts. We define an SBG according to Weedman et al. (1981) and avoid discussing Clumpy Irregulars and Extragalactic HII Regions. Second, the highest level of a nonstellar activity is found in the innermost pc of an active galactic nucleus (AGN). It is associated with the accretion process onto a supermassive black hole ( $\mathrm{SBH}$ ) presumably taking place in AGNs (Begelman, Blandford and Rees 1984; Rees 1984).

The AGN phenomenon is usually discussed within the context of the radiative and dynamical effects it exerts on the surrounding interstellar medium (ISM). Here we shall address the issue of how the stellar and gas dynamics in the host galaxy can trigger this activity in the first place. To the extent that internal dynamics of the host itself is likely to depend on tidal interactions with its neighbors, the AGN and SBG phenomena spread over more than seven decades in radius. This explains the enormous difficulties one encounters when trying to model them.

For the discussion of the latest observational results see papers by Heckman and Kennicutt (these proceedings). 


\section{Fueling AGNs}

Assuming a mass-to-energy conversion efficiency $\epsilon \sim 0.1$ for a typical AGN, the mass consumption rate can be estimated as

$$
\dot{M}_{A G N} \sim 2\left(\frac{\epsilon}{0.1}\right)^{-1}\left(\frac{L}{10^{46} \mathrm{erg} \mathrm{s}^{-1}}\right) \mathrm{M}_{\odot} \mathrm{yr}^{-1}
$$

If $\tau_{A G N}$ is the duty-cycle of a typical AGN (i.e. the total activity time), then approximately

$$
M_{\text {cycle }} \sim 2 \times 10^{9}\left(\frac{\epsilon}{0.1}\right)^{-1}\left(\frac{L}{10^{46} \mathrm{erg} \mathrm{s}^{-1}}\right)\left(\frac{\tau_{A G N}}{10^{9} \mathrm{yr}}\right) \mathrm{M}_{\odot}
$$

of gas must be at hand and confined to within

$$
r_{\text {cycle }} \lesssim 4 \alpha\left(\frac{T}{100 \mathrm{~K}}\right)\left(\frac{v_{\phi}}{200 \mathrm{~km} \mathrm{~s}^{-1}}\right)^{-1}\left(\frac{\tau_{A G N}}{10^{9} \mathrm{yr}}\right) \mathrm{pc}
$$

of the $\mathrm{SBH}$, if the extraction of angular momentum is done by a viscous torque in a geometrically thin accretion disk. Here $\alpha$ is defined by viscous stress $\sim \alpha \times$ internal pressure and is limited to $\alpha \lesssim 1$ for local viscosities, e.g., subsonic turbulence; $v_{\phi}$ is the local Keplerian velocity; and $T$ is the equatorial temperature of the disk. The equilibrium temperature in a dusty AGN disk beyond few pc will not exceed $T \sim 100 \mathrm{~K}$ even if $\sim 10 \%$ of the central radiation flux is backscattered from high lattitude (Shlosman and Begelman 1987, 1989; Phinney 1989). The formula (3) demonstrates the inefficiency of viscous torques in coupling the region beyond $r_{\text {cycle }}$ to the vicinity of the SBH. The fuel must be stored locally, within $r_{c y c l e}$, or some other mechanism should be considered for redistributing the angular momentum. As we intend to show below, the locally stored fuel cannot be in the form of stars and there is a real problem to keep it in a gaseous form for many dynamical times.

\section{Compact Star Clusters}

"Local" sources, usually a dense stellar cluster with a core radius of $R_{c l} \sim$ few pc and a mass $M_{c l}$, have been considered repeatedly in the 1970s. Several mechanisms have been proposed in order to convert the stars in the cluster into a 'digestible' form of fuel. They been reviewed recently by Shlosman, Begelman and Frank (1990). Here we only give the main points. First, a normal stellar evolution produces only $\sim 10^{-11} \mathrm{M}_{\odot} \mathrm{yr}^{-1} \mathrm{M}_{\sigma}^{-1}$ by means of the stellar mass loss. Second, stars can be tidally disrupted by the SBH, if the latter is less massive than $\sim 10^{8} \mathrm{M}_{\odot}$ (Hills 1975; Frank and Rees 1976; Lightman and Shapiro 1977). The resulting conversion rate of stars into gas is given by

$$
\dot{M}_{T D} \sim 2 \times 10^{-5}\left(\frac{m_{*}}{1 \mathrm{M}_{\odot}}\right)\left(\frac{M_{c l}}{10^{9} \mathrm{M}_{\odot}}\right)^{1 / 2}\left(\frac{R_{c l}}{10 \mathrm{pc}}\right)^{-3 / 2} \mathrm{M}_{\odot} \mathrm{yr}^{-1}
$$


where $m_{*}$ is the mass of a typical star in the cluster. Clearly such a low rate cannot support even moderate AGN luminosities unless the average cluster density is pushed into collisional regime. Third, the collisions among the cluster stars with dispersion velocities $\sigma_{*}$ will release

$$
\dot{M}_{\text {coll }} \sim \dot{M}_{T D}\left(\frac{\sigma_{*}}{v_{*}}\right)^{4},
$$

where $v_{*}$ is the escape velocity from a star. Apart from implicitly assuming that all tidally and collisionally produced stellar debris are accreted by the SBH, extremely compact star clusters are needed to support such high velocity dispersion. Fourth, hydrodynamical ablation of the cluster stars becomes relevant only when the cluster is close to a collisional regime and when the direct gas dynamical processes in the ISM are dominant. The ablation rate is limited to

$$
\dot{M} \sim\left(\frac{\rho}{\rho_{\text {cl }}}\right) \dot{M}_{\text {coll }}
$$

where $\rho$ is the density of the cluster ISM and $\rho_{c l}$ is the distributed stellar density in the cluster. Fifth, radiative ablation of stars by the nonthermal AGN continuum was considered in a number of works (Edwards 1980; Shull 1983; Voit and Shull 1988; Norman and Scoville 1988; Scoville and Norman 1988). Irradiating main sequence stars will have a negligible effect on their mass loss. Moreover the main sequence lifetime is prolonged. Thus, although red giants will suffer increased mass loss rates, this evolution phase will be delayed. Unless $\tau_{A G N}$ exceeds $10^{\circ} \mathrm{yrs}$, which seems to be highly unlikely (Padovani, Burg and Edelson 1990), the total mass loss will be reduced (Tout et al 1989).

The angular momentum problem is minimized in this class of "local" models, but it cannot be neglected. A combination of angular momentum and other constraints makes it difficult to obtain mass supplies adequate to fuel luminous AGNs. Star clusters of the requisite density and compactness have never been observed, and no compelling theoretical arguments demand their existence. As an alternative, we consider the ISM which is distributed throughout the main body of the host galaxy and is largely supported by its orbital motion.

\section{Geometrically Thin Accretion Disks, Thick Accretion Flows, and all that}

If the extraction of angular momentum is done by a viscous torque in a geometrically thin accretion disk, then $M_{c y c l e}$ must be confined to within the nucleus (in the form of gas), because of inefficiency of viscous drag. Such an accumulation of a gas will probably dominate the background gravitational potential and will violate the condition of a continuous disk. The latter will happen because accretion rates in excess of

$$
\dot{M}_{\text {crit }} \sim \frac{3 \alpha c_{s}^{3}}{G} \sim 5 \times 10^{-4} \alpha\left(\frac{T}{100 \mathrm{~K}}\right)^{3 / 2} \mathrm{M}_{\odot} \mathrm{yr}^{-1}
$$


lead to a locally gravitationally unstable disk and to a fragmentation. Here $c_{s}$ is the sound speed in the disk. The outcome to this instability in the gaseous disks depends upon the preferred equation of state: (i) the Jeans instability will saturate and the individual fragments will preserve finite cross sections and will interact by direct cloud-cloud collisions (Lin and Pringle 1987). An efficient cooling mechanism, e.g. high rate of collisions, will drive the disk deeper into domain of instability. This will allow for a whole range of unstable wavelengths, for an efficient transport of angular momentum outwards and for an inflow. (ii) Alternatively, the Jeans instability will proceed into nonlinear regime and as far as the resulting fragments are optically thin, they will contract to a point where they hardly interact at all and the disk will resemble more a stellar one than a turbulent gaseous disk (Shlosman and Begelman 1987). No substantial inflow is expected in this case.

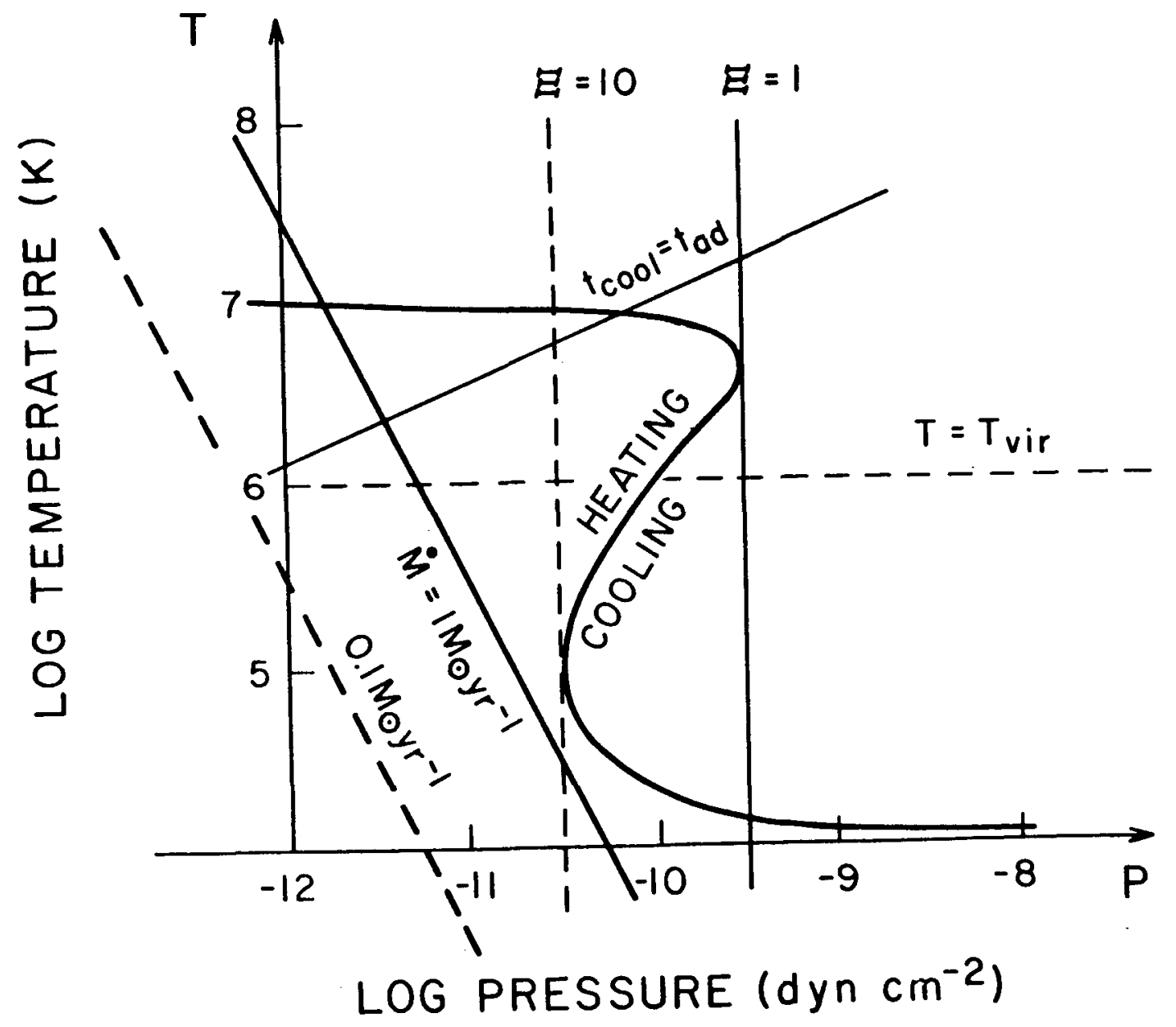

Fig. 1. Curves of a constant $\dot{M}=1$ and $0.1 \mathrm{M}_{\odot} \mathrm{yr}^{-1}$ on the $p-T$ plane for $\alpha=v_{\phi} / 100 \mathrm{~km} \mathrm{~s}^{-1}=r / 1$ kpc $=1$. The horizontal line labeled " $T=T_{v i r}$ " indicates the virial temperature above which the gas will tend to escape from the gravitational well, rather than accrete. Also plotted is the curve " $t_{\text {cool }}=t_{a d}$ ", below which radiative cooling dominates over the compressional heating associated with the accretion flow. The threshold for runaway heating is shown schematically, assuming an ionizing luminosity of $10^{45} \mathrm{erg} \mathrm{s}^{-1}$ and an AGN spectrum with an inverse Compton temperature of $10^{7} \mathrm{~K}$ (from Begelman, Frank and Shlosman [1989]). 
To avoid fragmentation in a continuous disk a stable solution with $\alpha>>1$ and/or $T>100 \mathrm{~K}$ must be found. Shlosman and Begelman (1989) and Begelman, Frank and Shlosman (1989) concluded that both are unacceptable and different ways of heating up the disk, including winds from $\mathrm{T}$ Tauri and $\mathrm{OB}$ stars and supernovae (SN), are inefficient. An alternative way to preserve the continuous disk is to assume that the flow is being hot, $T \gg>10^{4} \mathrm{~K}$, from the beginning. However, under a wide range of parameters, some external source of heating is required to prevent such a flow from catastrophic cooling and collapsing to a galactic plane. This is a consequence of high thermal pressure, 2-3 magnitudes above typical ISM pressure, required in order to obtain the fueling rates for luminous AGNs without exceeding the virial temperature (Begelman, Frank and Shlosman 1989 and Figure 1). Both the heating by SN blast waves and the X-ray continuum from the central source are more likely to cause outflow (galactic wind) than promote steady accretion. The possibility that cooling flows fuel at least some AGNs, however, cannot be ruled out at present.

We conclude that a thin homogeneous accretion disk is not a viable means of delivering fuel to luminous AGNs on scales much larger than a pc because of a long inflow time scale and effects of self-gravity, which lead to fragmentation and possibly star formation. There are also serious obstacles to maintaining and regulating the energetics of geometrically thick, hot accretion flows. However, if the disk is not required to be quasi-continuous, one can draw a straightforward analogy between a standard accretion disk and the disk of discrete particles, which has been described in connection with Saturn's rings (Begelman, Frank and Shlosman 1989 and refs. therein). An alternative approach, using dynamically important magnetic fields to drive accretion in the inner few tens of $\mathrm{pc}$ from the $\mathrm{SBH}$, has been recently reviewed by Blandford (1989).

\section{Fueling Starbursts}

Luminous starburst galaxies show SFRs that exceed the Galactic rate by two orders of magnitude or more. Most of it is confined to the inner kpc. High SFRs correlate with the amount of molecular gas (estimated from $\mathrm{CO}$ observations) concentrated in the same volume. The latter may correspond to about $50 \%$ of the total molecular gas in the galaxy (Sanders et al. 1988) and more than $\sim 10-20 \%$ of the dynamical mass within few hundred pc (e.g., Ishizuki et al. 1990). However, even $M_{\text {gas }} \sim 10^{9}-f e w \times 10^{10} \mathrm{M}_{\text {:) }}$ of gas will be exhausted in $\tau_{\text {burst }} \sim 10^{7} \mathrm{yrs}$ and locked in the low-mass stars (using Salpeter's IMF). Maintaining this SFR over a cosmological time would contradict rotation curves of disk galaxies. Even with a truncated IMF (at $\sim 3 \mathrm{M}_{\odot}$ ), $\tau_{\text {burst }}$ cannot be prolonged above $\sim 10^{7}-10^{8}$ yrs, because the gas would be heated up by SN and flow out in a wind (Chevalier and Clegg 1985).

The lifetime of a molecular cloud can hardly exceed $\sim 10^{8}$ yrs. It would be difficult, 
therefore, to explain a slow accumulation of a molecular gas to high densities and to dynamically important masses unless they are injected into the inner kpc on the comparable time scale. Injection rates $\sim 100 \mathrm{M}_{\odot} \mathrm{yr}^{-1}$ would satisfy the above requirement. The cloudcloud collision time scale is given by

$$
\tau_{\text {coll }} \sim \frac{t_{\text {orb }}}{C}
$$

where $\mathrm{C}$ is the cloud covering factor and $t_{\text {orb }}$ is the cloud orbital time at radius $r$. If the gaseous disk is supported vertically by the cloud random motion,

$$
C \sim 40\left(\frac{a_{c l}}{30 \mathrm{pc}}\right)^{2}\left(\frac{r}{500 \mathrm{pc}}\right)^{-2}\left(\frac{M_{g a s}}{10^{9} \mathrm{M}_{\odot}}\right)\left(\frac{M_{c l}}{10^{5} \mathrm{M}_{\odot}}\right)^{-1}
$$

where $a_{c l}$ and $M_{c l}$ are the cloud size and mass, chosen to be similar to the Milky Way clouds. It would be difficult to decrease $C$ substantially below unity. If massive star formation is simply related to molecular cloud collisions (Scoville, Sanders and Clemens 1986), eq.(9) results in $\tau_{\text {burst }} \sim \tau_{\text {coll }} \lesssim t_{\text {orb }}$. However, massive star formation in the disk may be initially suppressed and exhibit a threshhold behavior. Such a threshold may be the consequence of a velocity-dependent efficiency of massive star formation (e.g., Smith 1982). Suppose the clouds are injected with velocities which are of the order of their orbital velocities and not necessarily confined to the galactic plane. These velocities will be "thermalized" in cloud-cloud collisions and the gas will continue to accumulate until the collisions will reduce the dispersion velocities, flatten the gas distribution towards the plane and the runaway star formation will follow. Another possibility is that clouds injected into inner kpc are made of atomic and not molecular hydrogen. Collisions between $H I$ clouds do not result in gravitationally unstable configurations, do not lead to star formation but instead may induce the formation of $\mathrm{H}_{2}$ clouds (Stone 1970; Tubbs 1982). Only when the latter become abundant, the massive star formation will be triggered.

We conjecture that both luminous AGNs and SBGs depend upon an external reservoir of fuel and its efficient channeling to the inner pc and kpc respectfully. Hence the problem which must be addressed at this point is one of redistribution of the angular momentum in the gaseous component of the disk galaxy. Clearly it will impose much more stringent conditions on AGNs than on SBGs.

\section{Disk Galaxies: Angular Momentum Transfer}

The origin of starburst and nuclear activity in galaxies appears to be intimately related to the problem of global stability of these galaxies. Understanding how disks respond to different perturbations will clarify what triggers are most efficient in exciting particular modes. The goal is to find a mechanism that efficiently transfers gas between different 
parts of the stellar disk, i.e. from large radii to small radii, and is doing this on a time scale comparable with the dynamical time in the disk.

Radial flows are not expected to occur in an axially symmetric galaxy under normal circumstances. Fortunately, galaxies are not completely axisymmetric and possess spiral arms, oval distortions, bars, lenses, warps, etc. We shall assume, for simplicity, that a bi-symmetrical disturbance of amplitude $\delta \Sigma$ is present within radius $r$ in the otherwise homogeneous disk of density $\Sigma$. The $m=2$ mode, where $m$ is the azimuthal number, is preferred by Nature in building disk galaxies, which most frequently have bars and two spiral arms. Such a disturbance will impose a gravitational torque $T \sim G(\delta \Sigma)^{2} r^{3}$ on the test particle outside $r$. Let the disk with the mass $M_{d}(r)$ be embedded in a massive halo, $M_{h}(r)$, and let $M_{h}(r) / M_{d}(r)>1$, to ensure global stability at all relevant radii. The angular momentum within $r$ is $J(<r) \sim r^{3} \Sigma v_{\phi}$ and the time scale for extraction of this momentum is of the order of

$$
\tau_{J} \sim \frac{J}{T} \sim\left(\frac{M_{h}}{M_{d}}\right)\left(\frac{\Sigma}{\delta \Sigma}\right)^{2} t_{d y n}
$$

The above estimate was made more rigorously by Lynden-Bell and Kalnajs (1972) and Larson (1984). The eq.(10) underlines the dramatically short timescale of angular momentum transfer in self-gravitating disks when a mechanism for excitation and subsequent driving of non-axisymmetric perturbation exists. Because of the nature of a stellar "fluid", the growth rate of gravitational instability will probably saturate in the mildly nonlinear regime, i.e. $\delta \Sigma / \Sigma \lesssim 1$, but even $\sim 10 \%$ addition of cold gas may result in shocks and dissipation. The gas can be easily driven into a strongly nonlinear regime.

Contrary to the common belief, axisymmetric disturbances can redistribute angular momentum on the large scale as well. In order to understand this let us define the threshold for the onset of these instabilities in a mildly self-gravitating disk (Toomre 1964; Goldreich and Lynden-Bell 1965a):

$$
Q=\frac{c_{s} \kappa}{\pi G \Sigma},
$$

where $c_{s}$ is the sound speed in a homogeneous disk or a dispersion velocity in a disk of clouds or stars; $\kappa$ is the epicyclic frequency; and $\pi$ should be replaced by 3.36 for a stellar disk. A geometrically thin disk will be unstable for values of $Q<1$ and will fragment into clumps of the size of the disk thickness. This is the local Jeans instability discussed earlier in section 2 . The smallest wavelength which is still unstable is given by $\lambda_{\min } \sim c_{s}^{2} / G \Sigma$ and the largest unstable one is $\lambda_{\max } \sim G \Sigma / \Omega^{2}$, where $\kappa \sim \Omega$, the local angular velocity is assumed. The instability manifests itself in the local compression (i.e. heating) and mass transfer. The latter is always accompanied by an angular momentum transfer. One can define an effective kinematic viscosity $\nu_{e f f}$ which is responsible for such a transfer. Lin and Pringle (1987) gave a prescription to build $\nu_{e f f}$, assuming that the longest unstable 
wavelengths are the most efficient ones for $\mathrm{J}$-transfer and the characteristic time scale is the dynamical time in the disk:

$$
\nu_{e f f} \sim \lambda_{\max }^{2} / t_{\text {orb }}
$$

The resulting 'viscous' (inflow) timescale in the disk is:

$$
t_{\text {vigc }} \sim \frac{r^{2}}{\nu_{e f f}} \sim \frac{1}{1-Q^{2}}\left(\frac{M_{h}}{M_{d}}\right)^{2} t_{\text {orb }}
$$

In the $\alpha$-disk notation this is equivalent to $\alpha \sim Q^{-2} \sim \lambda_{\max } / h$, where $h$ is the disk thickness and $Q<1$. Hence this means $\alpha>1$. However, because the longest wavelengths have the slowest growth rates, eq.(12) probably overestimates the effective viscosity while eq.(13) underestimates the inflow time. For the sake of completeness, we note that the growth of axisymmetric modes is always accompanied by the growth of non-axisymmetric modes of comparable $\lambda$.

\section{Extrinsic vs Intrinsic Driving: Triggers}

Mechanisms that excite large-scale non-axisymmetric modes in galactic disks can be divided into two groups: extrinsic and intrinsic. The former include tidal encounters between galaxies (flybys), orbital torques by their satellites and mergers. The latter include instabilities brought about by the evolution of the disk, e.g., infall, distortions built in by initial conditions (bars, general triaxiality). In the absence of a comprehensive theory of self-gravitating disks, a variety of empirical methods have been used to map out domains of stability and to study the onset and nonlinear evolution of non-axisymmetric dynamical instabilites. Numerical modeling has shown that tidal interactions between galaxies, both flybys and mergers, usually lead to transient bars. Furthermore, a tidal interaction reinforces an already existing bar, provided the collision is not head-on (Combes 1987) and triggers the bar instability in otherwise stable stellar disk (Gerin, Combes and Athanassoula 1990). This is achieved by inducing or moving the inner Lindblad resonance (ILR). But a bar, in fact, may be a long-lived and self-sustained configuration resulting from a global self-gravitational instability, or even be relic from galaxy formation epoch. $N$ body simulations of collisionless stellar disks have revealed a strong tendency for low-order modes, and particularly for a bar-like mode, to grow in the absence of ILRs and a hot massive halo component. That disk experiences period(s) of dynamical instability may explain why such a high fraction, $\sim 50 \%$, of disk galaxies is barred.

Spiral Waves, Bars and Ovals

In order to understand the specifics of interaction of the unstable mode with the rest of the stellar disk and especially with the gaseous component, we shall discuss it in some 
detail. As a first step, we shall address the stellar dynamical instabilities, which occur on scales $\lesssim 1 \mathrm{kpc}$, and their triggers. Gasdynamical instabilities within the central kpc are reviewed in section 6 .

Spiral waves may have a two-fold effect on the galactic disk: (i) they cause slow angular momentum transport and radial inflow; and (ii) they strongly amplify the SFR when sufficient amount of gas is present, i.e. if one accepts that star formation is related to spiral waves through formation of molecular gas. The formation rate of the latter is enhanced by large quantities of gas going into nonlinear self-gravitating regime.

The nonlinear response of the gas to the background gravitational potential of a spiral arm was studied by Lubow, Balbus and Cowie (1986). For $15 \%$ gas contribution to the surface density in equilibrium disk, it dominates the region of the spiral density wave by a factor of a few. The global stellar mode is centrally condensed as the instability has more time to amplify itself at smaller radii as a result of short dynamical times there. This means that the amplitude of a stellar wave increases towards the center, e.g., $\Sigma_{* 1} \propto r^{-3 / 2}$ (Lubow 1988), and so does the gas response. With such a centrally concentrated response, the subsequent star formation will be centrally peaked either. This model will naturally prefer starbursts to occur in the late-type spirals, where gas is more abundant and its density is raising towards the center.

However, some important details have been omitted in the above estimates: (i) the effect of the starburst on cloud velocity dispersions (input of energy by SN); and (ii) the damping of stellar waves by cloud-cloud collisions. Because the gas response to stellar wave will be phase-shifted, the gas will produce torque on the stellar wave. As a result the stellar wave growth rate will be saturated and ultimately both waves will be damped. Lubow (1986) estimates the damping time scale for our Galaxy to be $\lesssim 10^{9} \mathrm{yrs}$, comparable or even shorter than growth rate for corresponding spiral modes (Haass 1983). Therefore cloud-cloud collisions are capable of limiting spiral density wave amplitudes; (iii) the effect of ILRs has been neglected; and (iv) the WKBJ approximation used breakes down at small radii because $k r \sim 1$ there.

As we have mentioned above, spiral waves have some effect on inducing radial flow of gas and may drastically increase the SFR. Unfortunately, spiral structure cannot extend across ILR and OLR (outer Lindblad resonance). It may of course reappear inside the ILR. If a bar exists, the actual living space for spirals may be even smaller. It is also important to remember that the inflow is limited to within the corotation radius (CR) because the torque will change the sign there: inflow at $r<r_{C R}$ towards the ILR and outflow at $r>r_{C R}$ towards the OLR. If no ILR is present, the spiral structure may be terminated at the $Q$-barrier (i.e. where $Q$ exceeds unity). This typically occurs in the inner parts of stellar disks (Freeman 1987), at $\sim 1-2 \mathrm{kpc}$ and hence the outer spiral structure has no effect inside $\sim 1 \mathrm{kpc}$. 
Bars form spontaneously in a system with an excess of bulk motion (e.g., rotation) over random motion and "where it is not prohibited by the law" (e.g., Binney and Tremaine 1987). For a collisionless (stellar) disk a reliable measure of stability is the value of $t_{O P} \equiv$ $T /|W|$, and $t_{O P}>0.14$ is sufficient condition for a global dynamical instability (Ostriker and Peebles 1973) and bar formation. Here $T$ is the total rotational energy and $W$ is the integral gravitational energy of the system. More generally speaking, the physical reason for bar instability is a strong amplification of any leading disturbance in a sheared disk into a trailing disturbance (swing amplifier), which then propagates through the disk center (feedback) and appears as a leading one (Goldreich and Lynden-Bell 1965b; Toomre 1981). The best way to stabilize the disk is to cut the feedback by introducing ILR, by heating-up the inner disk $(Q>3)$, or by surrounding it with a hot and massive halo.

Bars can extend to corotation, but are not obliged to be so long. They are efficient drives of spiral structure which originates at the opposite ends of a bar (Schwarz 1984; Combes and Gerin 1985). What makes the stellar bar-like mode so attractive as a mechanism for inducing the radial flows is the strong response of the gas within the bar radius $r_{b a r}$. It triggers a pair of large-scale shocks, i.e. regions of enhanced cloud-cloud collisions. A bar-like mode typically has a radial wavelength comparable to the size of the system; it is a global mode. It couples efficiently between very different radii and by doing so provides $\alpha>>1$ (in the $\alpha$-notation of the viscosity). Within the bar radius, the gas shocks on the leading edge of the bar and, in the absence of ILR, flows inwards on the dynamical time scale. But even in the absence of ILR, the radial flow will not extend all the way to the galactic nucleus $(\sim 1-10 \mathrm{pc})$, as one can expect naively. The condition for the existense of leading shocks is violated at smaller radii and an order of magnitude estimate shows that the gas will probably accumulate at $\sim 0.1 r_{\text {bar }}$ (Shlosman, Frank and Begelman 1989).

The overwhelming majority of disk galaxies are explicitly non-axisymmetric, because they show spiral structure and oval distortions. It is clear both on kinematic (Bosma 1981) and photometric (Kormendy 1982) grounds that many unbarred galaxies contain oval disks with intrinsic equatorial axial ratios of $\sim 0.8$. Such ovals are entirely sufficient to produce the same inward gas flow as do bars. In fact, since all of the disk is oval while $\lessgtr 20 \%$ of a disk is in the bar, the potential of an oval disk is easily as non-axisymmetric as that of a barred galaxy. There are several important points regarding oval distortions in disk galaxies. First, oval galaxies do not look barred in visible light. Omitting such galaxies biases conclusions that Seyferts are not preferentially non-axisymmetric. For example, the classic Seyferts NGC 1068 and NGC 4151 are prototypical ovals. Second, oval disks are easy to recognize photometrically. Third, infrared images sometimes reveal bars underlying oval disks, as in NGC 1068 (Scoville et al. 1988). Once we recognize that oval disks exist and rearrange gas like the bars do, the correlation between galaxies that are non-axisymmetric and ones that show nuclear activity gets much cleaner. 
We shall be interested here only in the effects of flybys and mergers on the galactic disk, ignoring the fate of the companion where it is possible. For our purposes, the most important result of a tidal interaction is the excitation of transient non-axisymmetric modes in otherwise stable stellar disk. Tidal forcing was first proposed by Holmberg (1941) and then by Toomre (1969). Relatively slow and direct passage results in a nice trailing spiral structure which lasts of the order of the passage time $\sim f e w \times 10^{8} \mathrm{yrs}$, as was shown in a numerical simulation by Toomre and Toomre (1972). If only $\sim 1 \%$ forcing (ratio of tangential-to-radial force) is needed to drive the spiral wave (Toomre 1981), a mass ratio between the flyby and the main galaxy of $\sim 10^{-2}$ or larger is required, if no interpenetration is allowed. The earliest numerical simulations showed that the largest effect (in terms of disturbing individual orbits) the flyby has on the outer disk. However, it was realized subsequently, that this was an artifact of an insufficient dynamic range used in simulations. Only very slow flybys will have no net effect on the inner stellar disk, because the perturbation will be adiabatic there.

Because of a strongly nonlinear response of a perturbed galaxy only numerical simulations of interaction are capable of treating the problem in a self-consistent manner. The large dynamic range makes it very difficult to actually follow the gaseous and stellar response (Noguchi 1988a,b). Generally, a transient bar $\sim$ few kpc develops in a few rotation periods after the closest approach. It disappears after some time and then reappears again, but weaker. In the intermediate phase, the galaxy looks very much similar to amorphous galaxies described by MacKenty (1990).

The perturber affects the gaseous component of a primary galaxy by (i) a direct tidal torque, forcing the gas to deviate from original motion; and (ii) by perturbing the gravitational potential of the stellar component which in turn provides additional and long-lived torque on the gas (Noguchi 1988b). When the stellar background potential is frozen, only the outer parts of the galaxy are affected (gas gravity is neglected here). The tidal force of the perturber alone fails to induce the infall. When the self-gravity of the stellar disk is taken into account the spiral structure also develops in the inner parts of the disk (still on kpc-scale, as the resolution of the numerical model is $\sim \mathrm{kpc}$ ). Initially, the massive star formation closely follows the large-scale spiral pattern and later peaks within the bar radius. At later stages the gas (cloud) dynamics is influenced only by the potential of the stellar disk, whereas effect of the perturber is negligible. The inflow rate is not a monotonic function of time (same as the bar strength). Therefore the galaxy still can show recurrent starburst activity, even when perturber has already receeded. These "latency periods" and episodic nature of the inflow have been also suggested to explain the absense of Seyfert nuclei in strongly interacting galaxies and the fact that not all galaxies in the multiple systems are Seyferts (Byrd, Sundelius and Valtonen 1987). Note, however, that 
the inflow on a kpc-scale may be completely unrelated to the inflow on a much smaller $\sim 10-100$ pc-scale, as we shall argue in section 6 . It is worth mentioning that cloud-cloud collisions provide the necessary dissipation and when they are artificially switched off the behavior of gas is identical to that of a stellar "fluid".

Thus, transient stellar bars are typical products of tidal interactions. Even when initial conditions are unfavorable to bar formation, e.g., $v_{\phi}=$ const, they still show up in the simulations, but are not as dominant (Byrd et al. 1986; Thomasson 1987).

Mergers produce substantial dy namical effects on stellar disks which result in burst of star formation and radial flows. When a rotating disk experiences tidal forcing, the satellite galaxy will define the positions of major resonances in the disk. The pattern speed of the disturbance is fixed by the satellite angular velocity of revolution. The timescale to accrete a small satellite with the mass $M_{\text {pert }}$ is given by Quinn and Goodman (1986):

$$
t_{\text {in }} \sim 4 \times 10^{8}\left(\frac{v_{\phi}}{220 \mathrm{~km} \mathrm{~s}^{-1}}\right)\left(\frac{M_{\text {pert }}}{10^{9} \mathrm{M}_{\odot}}\right)^{-1}\left(\frac{d_{\text {pert }}}{10 \mathrm{kpc}}\right)^{2} \mathrm{yr}
$$

where $d_{\text {pert }}$ is the galactocentric distance to the perturber. About $10 \%$ of the disk mass, i.e. $\sim M_{L M C}$, can come from accretion of small satellites. This accretion process heats up the stellar disk. The ratio of the kinetic energy of the random motion in the plane to that along the $z$-axis tends towards $3: 1$. A further increase in this ratio is limited by buckling of the disk plane, which acts to reduce the velocity anisotropy - the hose-pipe instability (Toomre 1987). The heating of the disk is quite substantial, as can be seen from simulations by Hernquist and Quinn (1989). There is a serious doubt that such a disk will respond with a strong spiral in case the event will repeat itself, as stellar velocity dispersions never decrease.

Numerical simulations of merging galaxies (e.g. Hernquist and Quinn 1989) have shown that $m=2$ mode is the dominant mode of angular momentum transfer when the satellite is orbiting in a direct sense. In the case of a retrograde merger (Hernquist 1989), only $m=1$ resonance is possible and a single leading spiral arm results, as expected (Athanassoula 1978). The gas is channeled towards the inner kpc (400 pc is the resolution limit), where its dynamical importance is amplified by the ability to dissipate its random motion. The energy input into the gas by $\mathrm{SN}$ and stellar winds and other sources is neglected in the above simulations as well as the star formation processes. These are expected to alter dramatically the last stages of evolution of the gas distribution.

The sinking satellite will be subject to the tidal torque which eventually will disrupt it, if its average density becomes less than that of the cannibal galaxy. The distributed density of a small galaxy, i.e. a dwarf spheroidal (except M32-type elliptical, but these are rare), which dominate the faint end of the Schechter luminosity function is too low for the appreciable part of the satellite to penetrate the inner kpc (Saito 1979). The latter may 
be crucial for populating the lose-cone orbits by stars that will be ultimately captured by the SBH (Roos 1981). But, as in the case of other triggers, the main effect of a merger will be on the gas.

\section{Gas: Dynamical Effects}

As we have already discussed, large-scale departures from axial symmetry are quite efficient in triggering radial flows in the galactic ISM, which then start to accumulate within the central $\mathrm{kpc}$ in the form of a gaseous ring. While this process can ultimately lead to a starburst, angular momentum residing in the gas will still prevent it from crossing another two to three decades in radius, which is necessary for the standard viscous processes and/or magnetic torques to take over and feed the SBH.

The dynamical importance of gas at large $r$ is limited to within the spiral arms. In the circumnuclear regions, however, the gas may affect the global dynamics. A collisional disk of clouds is capable of dissipating its energy of random motions - a process that makes it relatively cold when compared with the stellar disk. As a result, the self-gravity of the gas and its dynamic response are greatly enhanced, and the disk becomes prone to global non-axisymmetric instabilities (Norman 1988; Shlosman, Frank and Begelman 1988; 1989). Future evolution of the gaseous distribution implies that it is the gravitational disturbance and not the cloud-cloud collisions that drives the inflow on scales $\sim 100 \mathrm{pc}$. Recent data suggest that molecular bars are common in Seyferts (Heckman, these proceedings).

Alternatively, Lin, Pringle and Rees (1988) have assumed that clouds remain on circular orbits in the inner $2 \mathrm{kpc}$ and this gaseous disk is only marginally stable, with $Q \sim 1-2$. A perturbation introduced by a tidal interaction penetrates down to $\sim 0.5 \mathrm{kpc}$. Once perturbed the clouds rapidly increase their collision rate and drive the gaseous disk away from marginal stability, causing an inflow. To get required $\dot{M}$, however, the inflow time must be short, and hence the gas amount must exceed $10 \%$ of dynamical mass (i.e. of $M_{h}$ in eq.[13]) in the inner $2 \mathrm{kpc}$. We note that global non-axisymmetric instabilities will develop under the same circumstances, but they will grow even for $Q>1$, when the former instability is suppressed.

\section{Conclusions}

We have outlined theoretical models for induced starburst and nuclear activity in disk galaxies. The dynamic range of the phenomena exceeds seven orders of magnitude in radius. The fueling of AGNs and to a lesser degree that of SBGs require sources in the main body of the host galaxy. Gravitational torques applied to the stellar disks on scales of $\sim 1-10 \mathrm{kpc}$ are capable of inducing radial flows in the ISM of disk galaxies. Thus, both intrinsic as well as extrinsic triggers may be responsible for the SBG activity. We find that a two-step approach is necessary to explain the AGN phenomenon. The crucial second step consists of self-gravitational instabilities in the gas on scales of $\sim 100 \mathrm{pc}$. 
ACKNOWLEDGMENTS. It is a pleasure to thank the organizers of this stimulating meeting. I am indebted to my long-time collaborators Mitch Begelman and Juhan Frank. This work was supported by NSF grant AST84-51725.

\section{References}

Athanassoula, E. Astron. Astrophys. 69, 395 (1978).

Begelman, M.C., Blandford, R.D. \& Rees, M.J. Rev. Mod. Phys. 56, 255 (1984).

Begelman, M.C., Frank, J. \& Shlosman, I. (1989) in Theory of Accretion Disks, ed. F. Meyer et al. (Dordrecht: Kluwer Acad. Publ.), p. 373.

Binney, J. \& Tremaine, S. (1987) Galactic Dynamics (Princeton: Princeton Univ. Press). Blandford, R.D. (1989) in Theory of Accretion Disks, ed. F. Meyer et al. (Dordrecht: Kluwer Acad. Publ.), p. 35.

Bosma, Astron. J. 80, 1825 (1981).

Byrd, G.G., Sundelius, B. \& Valtonen, M. Astron. Astrophys. 171, 16 (1987).

Byrd, G.G., Valtonen, M.J., Sundelius, B. \& Valtaoja, L. Astron. Astrophys. 166, 75 (1986).

Chevalier, R.A. \& Clegg, A.W. Nature 317, 44 (1985).

Combes, F. (1987) in Starbursts and Galaxy Evolution, eds. T.X. Thuan et al. (Editions Frontieres: Gif Sur Yvette), p. 313.

Combes, F. \& Gerin, M. Astron. Astrophys. 150, 327 (1985).

Edwards, A.C. Mon. Not. R. astr. Soc. 190, 757 (1980).

Frank, J. \& Rees, M.J. Mon. Not. R. astr. Soc. 176, 633 (1976).

Freeman, K.C. Ann. Rev. Astr. Ap. 25, 603 (1987).

Gerin, M., Combes, F. \& Athanassoula, E. Astron. Astrophys. , in press.

Goldreich, P. \& Lynden-Bell, D. Mon. Not. R. astr. Soc. 130, 97 (1965a).

Goldreich, P. \& Lynden-Bell, D. Mon. Not. R. astr. Soc. 130, 125 (1965b).

Haass, J. (1983) in Kinematics, Dynamics and Structure of the Milky Way, ed. W.L.H. Schuter (Dordrecht: Reidel), p. 283.

Hernquist, L. Nature 340, 687 (1989).

Hernquist, L. \& Quinn, P.J. (1989) in The Epoch of Galaxy Formation, eds. C.S.Frenk et al. (Dordrecht: Kluwer Acad. Publ.), p. 427.

Hills, J.G. Nature 254, 295 (1975).

Holmberg, E. Astrophys. J. 94, 385 (1941).

Ishizuki, S. et al. Astrophys. J., in press.

Kormendy, J. (1982) in Morphology and Dynamics of Galaxies, 12th Saas-Fee Course, eds.

L. Martinet \& M. Mayor (Geneva Observatory), p. 115.

Larson, R.B. Mon. Not. R. astr. Soc. 206, 197 (1984).

Lin, D.N.C. \& Pringle, J.E. Mon. Not. R. astr. Soc. 225, 607 (1987).

Lin, D.N.C., Pringle, J.E. \& Rees, M.J. Astrophys. J. 328, 103 (1988).

Lightman, A.P. \& Shapiro, S.L. Astrophys. J. 211, 244 (1977).

Lubow, S.H. Astrophys. J. (Letters) 307, L39 (1986).

Lubow, S.H. Astrophys. J. (Letters) 328, L3 (1988).

Lubow, S.H., Balbus, S.A. \& Cowie, L.L. Astrophys. J. 309, 496 (1986).

Lynden-Bell, D. \& Kalnajs, A.J. Mon. Not. R. astr. Soc. 157, 1 (1972). 
MacKenty, J.W. Astrophys. J. Supp. Ser., in press.

Noguchi, M. Astron. Astrophys. 201, 37 (1988a).

Noguchi, M. Astron. Astrophys. 203, 259 (1988b).

Norman, C. (1988) in Galactic \& Extragalactic Star Formation, eds. R.E. Pudritz \& M.

Fich (Dordrecht: Kluwer Acad. Publ.), p. 495.

Norman, C. \& Scoville, N. Astrophys. J. 332, 124 (1988).

Ostriker, J.P. \& Peebles, P.J.E. Astrophys. J. 186, 467 (1973).

Padovani, Burg, R. \& Edelson, R.A. (1990) preprint.

Phinney, E.S. (1989) in Theory of Accretion Disks, ed. F. Meyer et al. (Dordrecht: Kluwer Acad. Publ.), p. 457.

Quinn, P.J. \& Goodman, J. Astrophys. J. 309, 472 (1986).

Rees, M.J. Ann. Rev. Astr. Ap. 22, 471 (1984).

Roos, N. Astron. Astrophys. 104, 218 (1981).

Saito, M. Pub. Astr. Soc. Japan 31, 181 (1979).

Sanders, D.B. et al. Astrophys. J. 325, 74 (1988).

Schwarz, M.P. Mon. Not. R. astr. Soc. 209, 93 (1984).

Scoville, N., Matthews, K., Carico, D.P. \& Sanders, D.B. Astrophys. J. (Letters) 327 , L61 (1988).

Scoville, N. \& Norman, C. Astrophys. J. 332, 163 (1988).

Scoville, N., Sanders, D.B. \& Clemens, D.P. Astrophys. J. (Letters) 310, L77 (1986).

Shlosman, I. \& Begelman, M.C. Nature 329, 810 (1987).

Shlosman, I. \& Begelman, M.C. Astrophys. J. 341, 685 (1989).

Shlosman, I., Begelman, M.C. \& Frank, J. Nature, in press.

Shlosman, I., Frank, J. \& Begelman, M.C. (1988) in Active Galactic Nuclei, eds. D. Osterbrock \& J. Miller (Kluwer Acad. Publ.), p. 462.

Shlosman, I., Frank, J. \& Begelman, M.C. Nature 338, 45 (1989).

Shull, J.M. Astrophys. J. 264, 446 (1983).

Smith, J. Astrophys. J. 238, 842 (1982).

Stone, M.E. Astrophys. J. 159, 293 (1970).

Thomasson, M. (1987) in The Few Body Problem, IAU Colloq. 96, ed. M. Valtonen (Dordrecht: Reidel).

Toomre, A. Astrophys. J. 139, 1217 (1964).

Toomre, A. Astrophys. J. 158, 899 (1969).

Toomre, A. (1981) in Normal Galaxies, eds. S.M. Fall \& D. Lynden-Bell (Cambridge Univ. Press), p. 111.

Toomre, A. (1987) private communication.

Toomre, A. \& Toomre, J. Astrophys. J. 178, 623 (1972).

Tout, C.A., Eggleton, P.P., Fabian, A.C. \& Pringle, J.E. Mon. Not. R. astr. Soc. 238, 427 (1989).

Tubbs, A.D. Astrophys. J. 255, 458 (1982).

Voit, G.M. \& Shull, J.M. Astrophys. J. 331, 197 (1988).

Weedman, D.W. it et al. Astrophys. J. 248, 105 (1981). 
Roos: I agree that dwarfs possibly do not survive in the central regions of bright galaxies. Also, their mass is so low that it probably takes more than a Hubble time for dynamical friction to drag them into the central region. The nuclei of small galaxies of 0.1L* however may be significantly dense to survive and to penetrate into the nucleus of a larger galaxy, especially if they contain a massive black hole.

Shlosman: I agree, but the effect on the stellar disk of the 'cannibal' will be amplified as well. This merger will probably lead to an elliptical galaxy. I find it difficult to reconcile with the fact that seyferts are disk galaxies.

Balsara: I have a comment about your comment on testing the continuum limits of SPH. Myself along with Norman, Bickness and Gingold have done a lot of work to show that it can be done in a multidimensional context. More recently I have carried out the full Von Neumann stability analysis of SPH and shown that $\Gamma_{0}, \Gamma^{+}$and $\Gamma^{-}$characteristics are very well propagated by SPH and also that the advection is in fact substantially superior to

finite difference. You do have to incorporate all the results of my analysis to get this good behavior though! 